\title{
Erratum: Supersensitive Ras activation in dendrites and spines revealed by two-photon fluorescence lifetime imaging
}

Ryohei Yasuda, Christopher D Harvey, Haining Zhong, Aleksander Sobczyk, Linda van Aelst \& Karel Svoboda Nature Neuroscience 9, 283-291(2006)

In the print version of this article and the version initially published online, the second equation under the heading 'Generation of color coded images' (in Methods) contained an error. The equation should read

$$
<\tau>\sim \frac{\int d t \cdot t \sum_{i} P_{i} \cdot \exp \left(-t / \tau_{i}\right)}{\int d t \cdot \sum_{i} P_{i} \cdot \exp \left(-t / \tau_{i}\right)}=\frac{\sum_{i} P_{i} \tau^{2}{ }_{i}}{\sum_{i} P_{i} \tau_{i}}
$$

The error has been corrected in the HTML and PDF versions of the article. This correction has been appended to the PDF version.

CORRIGENDUM

Corrigendum: Activity level controls postsynaptic composition and signaling via the ubiquitin-proteasome system

Michael D Ehlers

Nature Neuroscience 6, 231-242 (2003)

In the supplementary information initially published online to accompany this article, some values given in Supplementary Tables 1 and 2 were incorrect. The errors have been corrected online. This correction has been appended to the PDF version. 